\title{
Turbulent Dynamos and Magnetic Helicity
}

\author{
Hantao Ji \\ Princeton Plasma Physics Laboratory, Princeton University, P.O. Box 451, Princeton, NJ 08543
}

(April 29, 1999)

\begin{abstract}
It is shown that the turbulent dynamo $\alpha$-effect converts magnetic helicity from the turbulent field to the mean field when the turbulence is electromagnetic while the magnetic helicity of the mean-field is transported across space when the turbulence is electrostatic or due to the electron diamagnetic effect. In all cases, however, the dynamo effect strictly conserves the total helicity except for a battery effect which vanishes in the limit of magnetohydrodynamics. Implications for astrophysical situations, especially for the solar dynamo, are discussed.
\end{abstract}

PACS numbers: 52.30.Jb, 96.60.Hv, 91.25.Cw 
Magnetic fields are observed to exist not only in the planets and the stars [1] but essentially everywhere in the universe, such as the interstellar medium in galaxies and even in clusters of galaxies [2]. The origin of these cosmical magnetic fields has been explained mainly by dynamo theory [3], which is one of the most active research areas across multiple subdisciplines of physics. In particular, generation of an electromotive force (EMF) along a mean field by turbulence, or the well-known $\alpha$ effect [4], is an essential process in amplifying large-scale magnetic fields [5]. Experimentally, the $\alpha$ effect has been observed in toroidal laboratory plasmas [6].

Recently, there has been growing awareness that a topological constraint on the observed magnetic field, the conservation of magnetic helicity, may play an important role in solar flare evolution [7]. This follows the success of Taylor in explaining the observed magnetic structures in laboratory plasmas by conjecturing the same constraint during relaxation [8,9]. Magnetic helicity, a measure of the "knottedness"and the "twistedness" of magnetic fields [10,11], is closely related to the dynamo effect. Indeed, the $\alpha$ effect drives parallel current which twists up the field lines, thus increasing magnetic helicity on large scales. As a matter of fact, almost all the observed large scale cosmical poloidal (or meridional) magnetic fields, either in their dipolar or quadrupolar forms, have linkage with strong toroidal (or azimuthal) fields, leading to finite magnetic helicity.

One simple yet important question arises: how exactly is magnetic helicity affected by the dynamo process? Can magnetic helicity of the large-scale field be created by the dynamo process or merely be transported across space? Motivated by Taylor's conjecture, early studies [12] showed that the $\alpha$ effect only transports helicity of the large-scale field across space without affecting the total helicity, as supported by laboratory measurements [13]. However, a contradicting conclusion was drawn in a recent study [14], which showed that the $\alpha$ effect locally converts helicity from the turbulent field to the mean field, as supported by statistical and numerical studies on inverse helicity cascading to large scales $[15,16]$. Answers to the questions raised by this contradiction are in demand since they would reveal the nature of the dynamo effects and clarify the effectiveness or limitations of the magnetic 
helicity concept in determining the evolution of solar and laboratory plasmas in which the the dynamo process plays a role.

In this Letter, it is shown that both conclusions, i.e. creation or transport of the largescale magnetic helicity by the $\alpha$ effect, are valid depending on the nature of the turbulence which drives the dynamo effect. When the turbulence is electromagnetic, the $\alpha$ effect converts helicity from the turbulent, small-scale field to the mean, large-scale field. On the other hand, when the turbulence is electrostatic or due to the electron diamagnetic effect, the $\alpha$ effect transports the mean-field helicity across space without dissipation. In all cases, however, the $\alpha$ effect strictly conserves the total helicity except for a battery effect which vanishes in the limit of magnetohydrodynamics (MHD). Implications for astrophysical situations, especially for the solar dynamo, are discussed.

In order to include other possible dynamo effects in a plasma, we revisit the mean-field electrodynamics [5] using the generalized Ohm's law (ignoring the electron inertial term) [17]

$$
\boldsymbol{E}+\boldsymbol{v} \times \boldsymbol{B}-\boldsymbol{j} \times \boldsymbol{B} / e n+\nabla P_{e} / e n=\eta \boldsymbol{j}
$$

where $n$ is the electron density and $P_{e}$ the electron pressure. Every quantity $x$ is divided into a mean part $\bar{x} \equiv\langle x\rangle$, averaged over ensembles or space, and a turbulent part $\tilde{x}$ : $x=\bar{x}+\tilde{x}$. Then the mean and turbulent versions of the Ohm's law become

$$
\begin{array}{r}
\overline{\boldsymbol{E}}+\overline{\boldsymbol{v}}_{e} \times \overline{\boldsymbol{B}}+\nabla \bar{P}_{e} / e n+\mathcal{E}=\eta \overline{\boldsymbol{j}} \\
\widetilde{\boldsymbol{E}}+\widetilde{\boldsymbol{v}}_{e} \times \overline{\boldsymbol{B}}+\left(\overline{\boldsymbol{v}}_{e}+\widetilde{\boldsymbol{v}}_{e}\right) \times \widetilde{\boldsymbol{B}}-\mathcal{E}+\nabla \widetilde{P}_{e} / e n=\eta \tilde{\boldsymbol{j}},
\end{array}
$$

where $\boldsymbol{v}_{i}\left(\boldsymbol{v}_{e}\right)$ is the ion (electron) flow velocity and the relations $\boldsymbol{v} \approx \boldsymbol{v}_{i}$ and $\boldsymbol{j}=e n\left(\boldsymbol{v}_{i}-\boldsymbol{v}_{e}\right)$ have been used. The mean electromotive force $(\mathrm{EMF}) \mathcal{E}$ is given by

$$
\mathcal{E}=<\widetilde{\boldsymbol{v}} \times \widetilde{\boldsymbol{B}}>-<\widetilde{\boldsymbol{j}} \times \widetilde{\boldsymbol{B}}>/ e \bar{n} \approx<\widetilde{\boldsymbol{v}}_{e} \times \widetilde{\boldsymbol{B}}>
$$

(Small battery effects such as $\left\langle\tilde{n} \nabla \widetilde{P}_{e}>/ e \bar{n}^{2}\right.$ are neglected; see discussions later.) We note that the appearance of $\boldsymbol{v}_{e}$ only on the RHS of Eq.(4) is consistent with Ohm's law being a force balance on electrons. 
The parallel component of $\mathcal{E}$, or the $\alpha$-effect [4], along the mean field is of interest:

$$
\begin{aligned}
& \mathcal{E} \cdot \overline{\boldsymbol{B}}=-<\left(\widetilde{\boldsymbol{v}}_{e} \times \overline{\boldsymbol{B}}\right) \cdot \widetilde{\boldsymbol{B}}> \\
& =<\widetilde{\boldsymbol{E}} \cdot \widetilde{\boldsymbol{B}}>+<\nabla \widetilde{P}_{e} \cdot \widetilde{\boldsymbol{B}}>/ e \bar{n}-\eta<\widetilde{\boldsymbol{j}} \cdot \widetilde{\boldsymbol{B}}>
\end{aligned}
$$

Here, Eq.(3) has been used and the last term on the RHS is small in highly conductive plasmas but has been treated as the back-reaction of the magnetic field on the dynamo process $[15,18]$. Thus, two possible turbulent $\alpha$-effects $[19,20,6]$ are identified. The first term $\langle\widetilde{\boldsymbol{E}} \cdot \widetilde{\boldsymbol{B}}\rangle$ represents the contribution to $\widetilde{\boldsymbol{v}}_{e}$ from the turbulent $\widetilde{\boldsymbol{E}} \times \overline{\boldsymbol{B}}$ drift which is a single fluid (MHD) effect (MHD dynamo), while the second term, $\left\langle\nabla \widetilde{P}_{e} \cdot \widetilde{\boldsymbol{b}}\right\rangle / e \bar{n}$, is the contribution from the turbulent electron diamagnetic drift $\nabla \widetilde{P}_{e} \times \overline{\boldsymbol{B}}$ which is an electron fluid effect in the two-fluid framework (diamagnetic dynamo). We note that although both MHD and diamagnetic dynamo effects have actually been detected in the laboratory [19,20], only the MHD dynamo effect has been studied in most dynamo theories and simulations.

In general, the electric field can be split further into a curl-free part and a divergence-free part, often called "electrostatic" and "electromagnetic", respectively: $\boldsymbol{E}=-\boldsymbol{\nabla} \phi-\partial \boldsymbol{A} / \partial t$ where $\boldsymbol{A}$ is the vector potential of the magnetic field $\boldsymbol{B}$ and $\phi$ is the electrostatic potential. Then the turbulent dynamo EMF, Eq.(5), can be rewritten as

$$
\begin{aligned}
\mathcal{E} \cdot \overline{\boldsymbol{B}}= & -<\nabla \widetilde{\phi} \cdot \widetilde{\boldsymbol{B}}>-<\frac{\partial \widetilde{\boldsymbol{A}}}{\partial t} \cdot \widetilde{\boldsymbol{B}}> \\
& +\frac{<\nabla \widetilde{P}_{e} \cdot \widetilde{\boldsymbol{B}}>}{e n}-\eta<\widetilde{\boldsymbol{j}} \cdot \widetilde{\boldsymbol{B}}>
\end{aligned}
$$

where the first three terms correspond to effects due to electrostatic, electromagnetic, and electron diamagnetic turbulence, respectively. We shall see below that the type of turbulence is crucial in assessing effects of dynamo action on the magnetic helicity.

The definition [21] of magnetic helicity [10] $K$ contained in a volume $V$ is given by $K=\int \boldsymbol{A} \cdot \boldsymbol{B} d V$ and its rate of change of the helicity is

$$
\frac{d K}{d t}=-2 \int \boldsymbol{E} \cdot \boldsymbol{B} d V-\int\left(2 \phi \boldsymbol{B}+\boldsymbol{A} \times \frac{\partial \boldsymbol{A}}{\partial t}\right) \cdot d \boldsymbol{S}
$$


where $V$ is enclosed by the surface $\boldsymbol{S}$. The integral under the volume integration represents the volume rate of change of helicity, while the integral under the surface integration represents flux of helicity across the surface. We note that only the volume integration term can possibly create or destroy helicity, and the surface integration terms merely transport helicity across space without affecting the total helicity. In the aforementioned mean-field electrodynamics, the mean helicity $\langle K\rangle$ is the sum of the helicity in the mean field, $K_{\mathrm{m}}=\int \overline{\boldsymbol{A}} \cdot \overline{\boldsymbol{B}} d V$, and the helicity in the turbulent field, $K_{\mathrm{t}}=\int\langle\widetilde{\boldsymbol{A}} \cdot \widetilde{\boldsymbol{B}}\rangle d V$. From Eq.(7), their rates of change are given by

$$
\begin{aligned}
\frac{d K_{\mathrm{m}}}{d t}= & -2 \int \overline{\boldsymbol{E}} \cdot \overline{\boldsymbol{B}} d V-\int\left(2 \bar{\phi} \overline{\boldsymbol{B}}+\overline{\boldsymbol{A}} \times \frac{\partial \widetilde{\boldsymbol{A}}}{\partial t}\right) \cdot d \boldsymbol{S} \\
\frac{d K_{\mathrm{t}}}{d t}= & -2 \int<\widetilde{\boldsymbol{E}} \cdot \widetilde{\boldsymbol{B}}>d V \\
& -\int\left(2<\widetilde{\phi} \widetilde{\boldsymbol{B}}>+<\widetilde{\boldsymbol{A}} \times \frac{\partial \widetilde{\boldsymbol{A}}}{\partial t}>\right) \cdot d \boldsymbol{S},
\end{aligned}
$$

where substitution of $\overline{\boldsymbol{E}}$ and $\widetilde{\boldsymbol{E}}$ by Eqs.(2) and (3) yields

$$
\begin{gathered}
\overline{\boldsymbol{E}} \cdot \overline{\boldsymbol{B}}=\eta \overline{\boldsymbol{j}} \cdot \overline{\boldsymbol{B}}-\mathcal{E} \cdot \overline{\boldsymbol{B}}+\nabla \cdot\left(\frac{\bar{P}_{e} \overline{\boldsymbol{B}}}{e n}\right) \\
<\widetilde{\boldsymbol{E}} \cdot \widetilde{\boldsymbol{B}}>=\eta<\widetilde{\boldsymbol{j}} \cdot \widetilde{\boldsymbol{B}}>+\mathcal{E} \cdot \overline{\boldsymbol{B}}+\nabla \cdot\left(\frac{<\widetilde{P}_{e} \widetilde{\boldsymbol{B}}>}{e n}\right) .
\end{gathered}
$$

The turbulent EMF appears as the second terms on the RHS of these equations but with opposite signs. It might be concluded that the dynamo effects would generate the same amount of helicity but with opposite signs in the mean field and the turbulent field [14]. However, this may not be the case depending on the nature of the turbulence.

By the use of Eq.(6), after cancellation and rearrangement of some terms, we obtain

$$
\begin{aligned}
\frac{d K_{\mathrm{m}}}{d t}= & -2 \int(\eta \overline{\boldsymbol{j}} \cdot \overline{\boldsymbol{B}}+\eta<\widetilde{\boldsymbol{j}} \cdot \widetilde{\boldsymbol{B}}>+\underbrace{\left.\frac{\partial \widetilde{\boldsymbol{A}}}{\partial t} \cdot \widetilde{\boldsymbol{B}}>\right)}_{A} d V \\
& -\int(\underbrace{2 \bar{\phi} \overline{\boldsymbol{B}}}_{B}-\underbrace{2 \frac{\bar{P}_{e} \overline{\boldsymbol{B}}}{e n}}_{C}+\underbrace{\overline{\boldsymbol{A}} \times \frac{\partial \overline{\boldsymbol{A}}}{\partial t}}_{D}+\underbrace{2<\widetilde{\phi} \widetilde{\boldsymbol{B}}>}_{E} \\
& -\underbrace{2<\frac{\tilde{P}_{e} \widetilde{\boldsymbol{B}}}{e n}}_{F}) \cdot d \boldsymbol{S}
\end{aligned}
$$




$$
\frac{d K_{\mathrm{t}}}{d t}=2 \int \underbrace{<\frac{\partial \widetilde{\boldsymbol{A}}}{\partial t} \cdot \widetilde{\boldsymbol{B}}>}_{A} d V-\int \underbrace{\left.<\widetilde{\boldsymbol{A}} \times \frac{\partial \widetilde{\boldsymbol{A}}}{\partial t}\right\rangle}_{G} \cdot d \boldsymbol{S} .
$$

A brief discussion will be useful here for each term of these equations. The term $D$ is responsible for the most common source of helicity for a toroidal laboratory plasma, in which a transformer supplies poloidal (toroidal) flux to be linked with existing toroidal (poloidal) flux. The term $B$ is responsible for the technique often called "electrostatic helicity injection" [22], in which a voltage is applied between two ends of a flux tube. The same amount of helicity with the opposite sign is also injected into the space outside the system, which is often a vacuum region [23]. The term $C$ has never been used to inject or change the helicity in a system. The term $G$ represents transport of helicity in turbulence by the propagation of electromagnetic waves possessing finite helicity (such as a circularly polarized Alfvén wave), without affecting the mean field helicity since term $A$ vanishes if there is no dissipation. Dissipation can result in a finite term $A$ which converts helicity from the turbulent field to the mean field [24] or vice versa [25].

The role of the turbulent dynamo in magnetic helicity evolution depends critically on the nature of the turbulence. In the case of electromagnetic turbulence, i.e. $\tilde{\boldsymbol{v}}_{e}$ is driven by an inductive electric field (which is divergence-free), the dynamo effect generates the same amount of helicity in both the mean and turbulent fields but with opposite signs, as seen from term $A$ of the above equations. In the case of electrostatic or electron diamagnetic turbulence, i.e. $\widetilde{\boldsymbol{v}}_{e}$ is driven by an electrostatic field or an electron pressure gradient (which are curl-free), the dynamo action does not affect the turbulent helicity but merely transports the mean-field helicity across space, as seen from the terms $E$ and $F$ in Eq.(8). We note that in order for terms $E$ and $F$ to have a net effect on the mean-field helicity, the electrons must be non-adiabatical, i.e. $e \tilde{\phi} / T_{e} \neq \tilde{n} / n$, a condition often satisfied in laboratory plasmas which are driven away from thermal equilibrium.

Despite the long history of the dynamo problem, there are no generally accepted theories on the nature of the turbulence which drives dynamo EMF's. It also has not been investigated numerically. Experimentally, however, it has been measured by probe techniques 
that the turbulence responsible for the observed $\alpha$-effect in laboratory Reversed-Field Pinch (RFP) plasmas is predominantly electrostatic [19] or electron diamagnetic [20]. In either case, the dynamo effect causes helicity transport in the mean field without effects on the turbulent field, which is consistent with theories [12]. Helicity transport due to the turbulent dynamo also has been verified experimentally [13]. Figure 1 shows an example of measured helicity flux induced by the electrostatic turbulence together with the measured $\alpha$-effect in an RFP plasma. Both measurements (thin lines) agree well with the predictions (thick lines) from the rest of the terms in Ohm's law and the helicity balance equation, indicating that the electrostatic turbulence alone is responsible for both dynamo action and helicity transport.

In the case of astrophysical dynamos, however, there is no observational evidence on the nature of the responsible turbulence. Such knowledge would have great implications on the role of dynamo action in the evolution of magnetic helicity. A good example under hot debate is the solar dynamo problem and its relationship with the observed twisted field lines (hence the helicity) on the solar surface [26,27] and even in the solar wind [7]. It has been found that one sign of the observed helicity appears preferentially on one hemisphere while the opposite sign appears preferentially on the other hemisphere. Naturally, an important question arises about the origin of this helicity preference. A generally accepted argument is that this helicity preference should originate from the convection zone or even a thin layer at the bottom of the convection zone where the solar dynamo is believed to be operational [28]. If the dynamo turbulence is electromagnetic, magnetic helicity in the large-scale field will be generated while leaving the same amount of helicity with the opposite sign in the small-scale turbulence, which may dissipate locally. On the other hand, if the turbulence is electrostatic or electron diamagnetic, the dynamo action will not affect helicity in the small-scale field but will transport or separate the large-scale helicity of one sign to one hemisphere while leaving the opposite helicity in the other hemisphere. After rising to the solar surface via the buoyancy force, these large-scale structures and its associated helicity are constantly removed from the sun by flaring. Both mechanisms, helicity conversion from the small 
scale to the large scale or helicity separation in the large scale from one hemisphere to the other, can replace the lost helicity continuously. However, the former mechanism conserves magnetic helicity locally in each hemisphere while both hemispheres need to be included for the latter mechanism to conserve helicity.

Despite the lack of theoretical insight, we point out here a general tendency in which plasma beta $\beta$, i.e. the ratio of plasma pressure force to magnetic force, may play an important role in determining the nature of the dynamo turbulence. In plasmas where $\beta \lesssim 1$, the turbulence is prone to be electrostatic or electron diamagnetic, which is consistent with laboratory measurements in RFP plasmas. Each field line can have a different electrostatic potential $\phi$ or electron pressure $P_{e}$ insulated by the strong magnetic field, leading to notable gradients in the perpendicular direction. On the other hand, in plasmas where $\beta \gg 1$, the turbulence becomes less electrostatic or electron diamagnetic due to diminishing magnetic insulation in the perpendicular direction and becomes more electromagnetic since the field lines tend to be pushed around by a much larger plasma pressure. This conjecture is supported by a general tendency of "reduction of dimensionality" [29], in which isotropic 3D turbulence reduces to anisotropic, 2D turbulence when a strong large-scale magnetic field is introduced.

In contrast to the low-beta plasmas in the laboratory, astrophysical plasmas with an active dynamo usually have a beta much larger than unity. In addition to the aforementioned solar dynamo, similar situations exist for cases of the geodynamo [30] and the galactic dynamo $[31,2]$. The aforementioned conjecture would predict a local conversion process of magnetic helicity by dynamo action from the turbulent field to the mean field. Both helicities contained in the turbulent and mean fields can be either dissipated due to a finite resistivity or transported out of the dynamo region through terms $D$ and $G$ in Eqs.(8) and (9). Clearly, a detailed study of the nature of the dynamo turbulence for each specific astrophysical situation is beyond the scope of this Letter, but it certainly warrants further investigations not only theoretically and numerically but also observationally and experimentally.

Regardless of the nature of the dynamo turbulence, the total helicity $K_{m}+K_{t}$ is always 
conserved as per Eqs.(8) and (9). This can be shown more rigorously by substituting the generalized Ohm's law Eq.(1) into the first term on the RHS of Eq.(7) to yield

$$
\int \boldsymbol{E} \cdot \boldsymbol{B} d V=\int \eta \boldsymbol{j} \cdot \boldsymbol{B} d V+\int \frac{\nabla P_{e} \cdot B}{e n} d V
$$

The first term on the RHS is not a dynamo effect but a resistive effect, which vanishes with zero resistivity. The second term on the RHS of Eq.(10) can be rewritten as $\int\left(\nabla P_{e}\right.$. $\boldsymbol{B} / e n) d V=\int\left(T_{e} / e\right) \boldsymbol{B} \cdot d \boldsymbol{S}+\int\left(T_{e} \boldsymbol{\nabla} n \cdot \boldsymbol{B} / e n\right) d V=\int\left(T_{e} / e\right)(1+\ln n) \boldsymbol{B} \cdot d \boldsymbol{S}-\int\left(\ln n \boldsymbol{\nabla} T_{e}\right.$. $B / e) d V$ for which both finite gradients in density and electron temperature (of course also in electron pressure) along the field line are necessary conditions to change the total helicity. However, we note that such parallel gradients, especially $\nabla_{\|} T_{e}$, are very small owing to fast electron flow along the field lines (with a few exceptions such as in laserproduced plasmas [32]). Such effects, often called the battery effect [1], provide only a seed for magnetic field to grow in a dynamo process and, of course, it can be accompanied by small but finite magnetic helicity. In the limit of MHD in which no parallel gradients allowed, dynamo action strictly conserves the total helicity. This result is consistent with the observation that the helicity is approximately conserved during magnetic relaxation [13] in the RFP plasmas.

In summary, it has been shown that the effect of turbulent dynamos on magnetic helicity depends critically on the nature of the turbulence. When the turbulence is electromagnetic, the $\alpha$ effect converts helicity from the turbulent, small-scale field to the mean, large-scale field. On the other hand, when the turbulence is electrostatic or due to the electron diamagnetic effect, the $\alpha$ effect transports the mean-field helicity across space without dissipation. Both mechanisms can explain the observed helicity preference of large-scale magnetic structures on the solar surface but they conserve helicity in different ways. Based on laboratory observations of turbulent dynamos in RFP plasmas, it is conjectured heuristically that plasma beta plays an important role in determining the nature of the turbulence; i.e. turbulent flow is driven by (curl-free) electrostatic electric field or electron pressure gradient when $\beta \lesssim 1$ and by (divergence-free) electromagnetic electric field when $\beta \gg 1$. In all 
cases, however, dynamo processes conserve total helicity except for a small battery effect which vanishes in the MHD limit. This is consistent with the observation that helicity is approximately conserved during laboratory magnetic relaxation. Detailed understanding of dynamo turbulence and its effects on magnetic helicity await further investigations not only by theories and numerical simulations but also by observations in space and well-controlled laboratory experiments.

The author is grateful to Dr. R. Kulsrud for his comments on gauge invariance, Dr. P. Diamond for his comments on the electron adiabatic response, and Dr. M. Yamada for valuable discussions. 


\section{REFERENCES}

[1] E.N. Parker, in Cosmical Magnetic Fields (Clarendon Press, Oxford, 1979).

[2] E.G. Zweibel and C. Heiles, Nature 385, 131 (1997).

[3] H.K. Moffatt, Magnetic Field Generation in Electrically Conducting Fluids (Cambridge University Press, 1978)

[4] E.N. Parker, Astro. Phys. J. 121, 293 (1955).

[5] F. Krause and K.-H. Rädler, in Mean-Field Magnetohydrodynamics and Dynamo Theory (Akademie-Verlag, Berlin, 1980).

[6] H. Ji et al., Phys. Plasmas 3, 1935 (1996).

[7] D.M. Rust, Geophys. Res. Lett. 21, 241 (1994).

[8] J.B. Taylor, Phys. Rev. Lett. 33, 1139 (1974).

[9] J.B. Taylor, Rev. Mod. Phys. 58, 741 (1986).

[10] L. Woltjer, Proc. Natl. Acad. Sci. USA 44, 489 (1958).

[11] M.A. Berger and G.B. Field, J. Fluid Mech. 147, 133 (1984).

[12] A.H. Boozer, J. Plasma Phys. 35, 133 (1986); E. Hameiri and A. Bhattacharjee, Phys. Fluids 30, 1743 (1987).

[13] H. Ji, S.C. Prager, and J.S. Sarff, Phys. Rev. Lett. 74, 2945 (1995).

[14] N. Seehafer, Phys. Rev. E 53, 1283 (1996).

[15] A. Pouquet, U. Frish, and J. Leorat, J. Fluid Mech. 77, 321 (1976).

[16] T. Stribling and W. H. Matthaeus, Phys. Fluids B 2, 1979 (1990).

[17] L. Spitzer, Jr., in Physics of Fully Ionized Gases (2nd Revised Edition) (Interscience Publishers, New York, 1962). 
[18] A.V. Gruzinov and P.H. Diamond, Phys. Rev. Lett. 72, 1651 (1994); A. Bhattacharjee and Y. Yuan, Astrophys. J. 449, 739 (1995).

[19] H. Ji et al., Phys. Rev. Lett. 73, 668 (1994).

[20] H. Ji et al., Phys. Rev. Lett. 75, 1085 (1995).

[21] When the volume $V$ is not singly connected or the magnetic field is not tangent to its surface, the definition of magnetic helicity needs to be modified (such as the introduction of relative helicity as described in Ref. 11) for gauge invariance. However, since the main conclusions do not depend on these variations, the simplest definition of magnetic helicity will be used in this Letter.

[22] J.B. Taylor and M.F. Turner, Nucl. Fusion 29, 219 (1989).

[23] A.H. Boozer, Phys. Fluids B 5, 2271 (1993).

[24] See, e.g. R.R. Mett and J.A. Tataronis, Phys. Rev. Lett. 63, 1380 (1989).

[25] Z. Yoshida and S.M. Mahajan, Phys. Rev. Lett. 72, 3989 (1994).

[26] D.M. Rust and A. Kumar, Solar Phys. 155, 69 (1994).

[27] A.A. Pevtsov, R.C. Canfield, and T.R. Metcalf, Astrophys. J. 440, L109 (1995).

[28] See, e.g. P.A. Gilman, C.A. Morrow, and E.E. DeLuca, Astrophys. J. 338, 528 (1989).

[29] G.P. Zank and W.H. Matthaeus, Phys. Fluids A 5, 257 (1993).

[30] See, e.g. G.A. Glatzmaier and P.H. Roberts, Science 274, 1887 (1996).

[31] R.M. Kulsrud, to appear in Annu. Rev. Astron. Astrophys. (1999).

[32] J. Stamper, Science 281, 1469 (1998). 


\section{FIGURES}
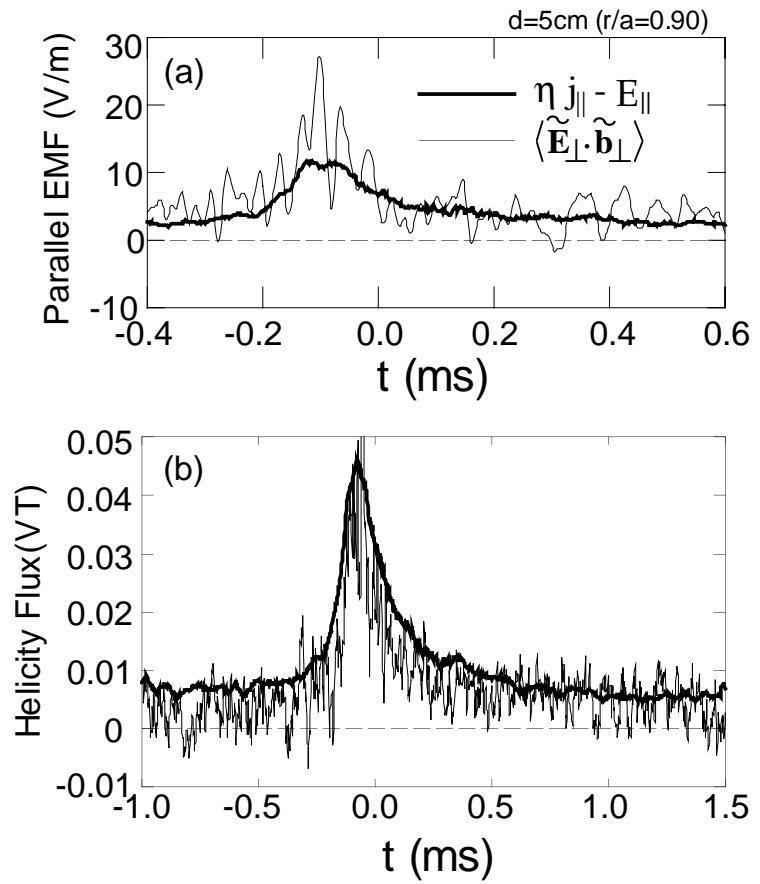

FIG. 1. Measured (a) parallel EMF ( $\alpha$-effect) due to electrostatic turbulence, $\left\langle\widetilde{\mathbf{E}}_{\perp} \cdot \widetilde{\mathbf{b}}_{\perp}\right\rangle$ (thin line) where $\widetilde{\mathbf{E}}_{\perp}=-\nabla_{\perp} \tilde{\phi}$, and (b) helicity flux (thin line) $<\widetilde{\phi} \widetilde{\mathbf{B}}>$ in a laboratory plasma (Ref. 6). The thick lines in both (a) and (b) are the predictions from the rest of the terms in Ohm's law and the helicity balance equation. The good agreements indicate that the electrostatic turbulence alone is responsible for both dynamo action and helicity transport. (The $t=0$ refers to the timing of magnetic relaxation events, during which both the $\alpha$-effect and helicity transport are enhanced over a constantly working turbulent dynamo effect.) 